\title{
Small Molecule Inhibitor of Mitotic Spindle Bipolarity Identified in a Phenotype-Based Screen
}

\author{
Thomas U. Mayer, ${ }^{1 *}$ Tarun M. Kapoor, ${ }^{1}$ Stephen J. Haggarty, ${ }^{2,3}$ \\ Randall W. King, ${ }^{2}$ Stuart L. Schreiber, ${ }^{2,3}$ Timothy J. Mitchison ${ }^{1,2}$
}

Small molecules that perturb specific protein functions are valuable tools for dissecting complex processes in mammalian cells. A combination of two phenotype-based screens, one based on a specific posttranslational modification, the other visualizing microtubules and chromatin, was used to identify compounds that affect mitosis. One compound, here named monastrol, arrested mammalian cells in mitosis with monopolar spindles. In vitro, monastrol specifically inhibited the motility of the mitotic kinesin Eg5, a motor protein required for spindle bipolarity. All previously known small molecules that specifically affect the mitotic machinery target tubulin. Monastrol will therefore be a particularly useful tool for studying mitotic mechanisms.

Cell-permeable small molecules can rapidly perturb the function of their targets and are therefore powerful tools to dissect dynamic cellular processes. However, such modulators are not available for most of the

1Department of Cell Biology, and 'Institute of Chemistry and Cell Biology, Harvard Medical School, Boston, MA 02115, USA. ${ }^{3}$ Howard Hughes Medical Institute, Department of Chemistry and Chemical Biology. and Department of Molecular and Cellular Biology. Harvard University, Cambridge, MA 02138, USA.

*To whom correspondence should be addressed. Email: Thomas_Mayer@hms.harvard.edu proteins involved in essential processes, and many of the ones that are available are nonspecific. The only known small molecules that specifically affect the mitotic machinery target tubulin ( 1 ), a subunit of the microtubules in the mitotic spindle. To identify cell-permeable small molecules that target other mitotic proteins, we developed the screening strategy in (Fig. 1A). First, we used the versatile whole-cell immunodetection (cytoblot) assay (2) to identify compounds that increase the phosphorylation of nucleolin. Nucleolin is a nucle- 
A

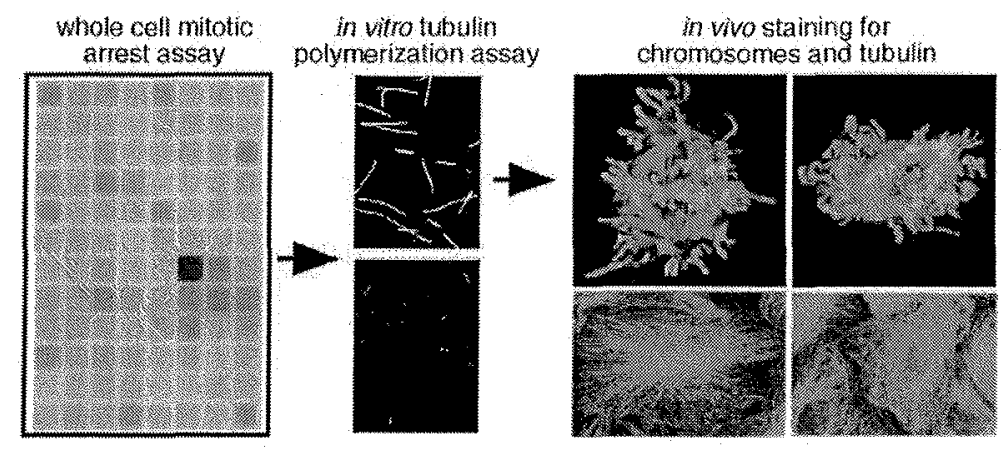

B

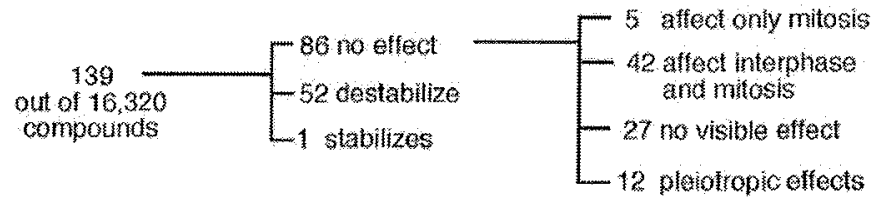

Fig. 1. Identification of five small molecules that affect mitosis. (A) From a whole-cell immunodetection assay, we selected 139 cell-permeable compounds that caused increases in phosphonucleolin staining in A549 cells (4). After molecules that target pure tubulin (5) were eliminated, the effect of the antimitotic compounds on microtubules (green), actin (not shown), and chromatin (blue) distribution was imaged (8). Examples of the effects of two different small molecules on BS-C-1 cells in mitosis (Upper) and in interphase (Lower) are shown. (B) Summary of screening results. Twelve antimitotic compounds tested on cells had pleiotropic effects and were not evaluated further.
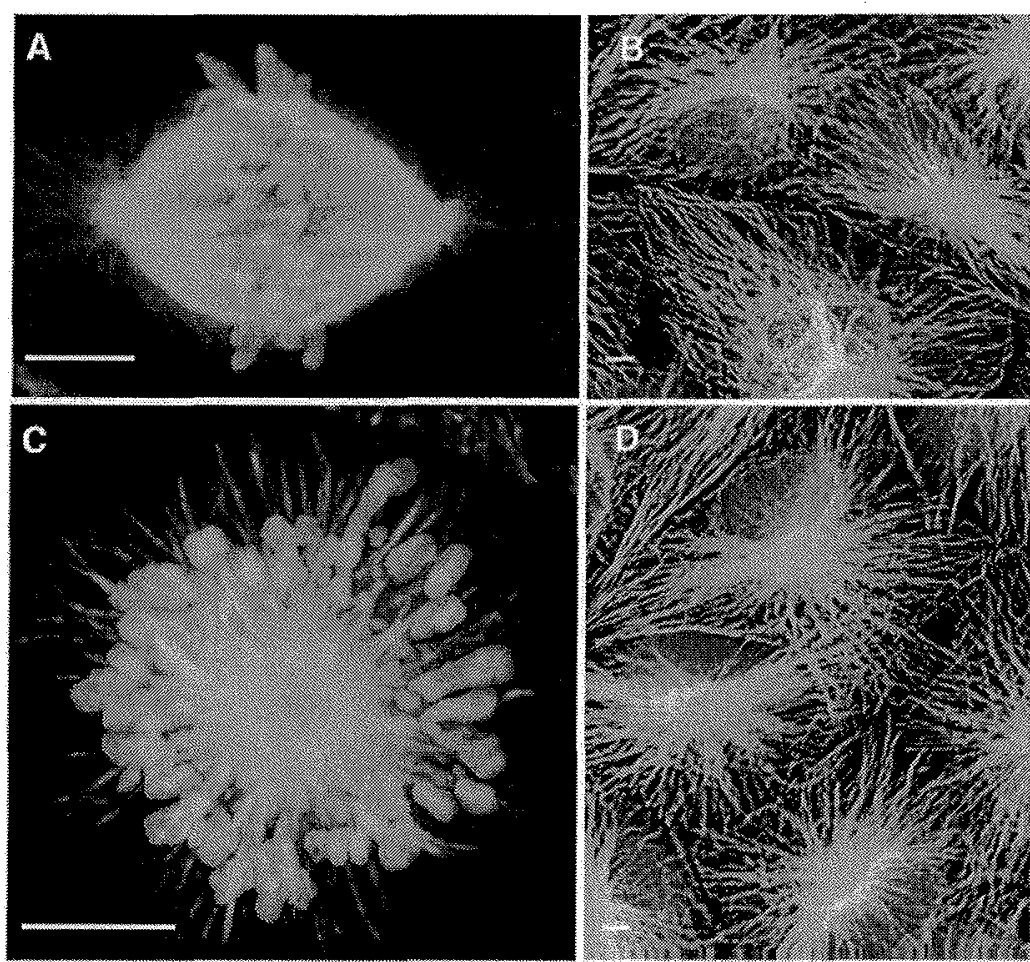

Fig. 2. Monastrol causes monoastral spindles in mitotic cells. Immunofluorescence staining [ $\alpha$-tubulin, (green), chromatin, (blue)] of BS-C-1 cells treated for 4 hours with $0.4 \%$ DMSO (control) $(A$ and $B)$ or $68 \mu \mathrm{M}$ monastrol (C and D). No difference in distribution of microtubules and chromatin in interphase cells was observed ( $B$ and D). Monastrol treatment of mitotic cells replaces the normal bipolar spindle (A) with a rosette-like microtubule array surrounded by chromosomes (C). Scale bars, $5 \mu \mathrm{m}$. olar protein that is specifically phosphorylated in cells entering mitosis, and compounds that cause mitotic arrest would be expected to show the phenotype of increased amounts of phosphonucleolin (3). Using this assay, we selected 139 compounds from a library of 16,320 small molecules (Fig. 1) (4).

Because many known antimitotics target tubulin, we tested each of the 139 compounds for the ability to affect tubulin polymerization in vitro (5). Fifty-two compounds inhibited tubulin polymerization (Fig. 1B) (6), and one stimulated tubulin polymerization, like the drug Taxol (7). The remaining 86 small molecules that increased the mitotic index presumably targeted other proteins involved in mitosis and were therefore studied further.

We treated mammalian epithelial kidney cells (BS-C-1) with each of the 86 remaining compounds and examined the distribution of microtubules, actin, and chromatin in fixed cells by fluorescence microscopy (8). Twenty-seven compounds had no observable effect on the microtubule and actin cytoskeleton or on chromosome distribution. There was an increase in the number of normal-appearing mitotic cells in response to these compounds, consistent with the observation that these compounds increase the amount of nucleolin phosphorylation (Fig. 1B). These compounds may increase the mitotic index by perturbing the function of proteins that regulate progression through the cell cycle, such as anaphase regulators, rather than structural or mechanochemical components of the mitotic spindle. It is also possible that these compounds have a subtle effect on cytoskeletal dynamics or chromosome organization that may not be observable in fixed cells.

Forty-two compounds affected cells in interphase as well as in mitosis (Fig. 1B). Cells treated with these small molecules had disorganized or partially depolymerized interphase microtubules in addition to abnormal mitotic spindle structures and misaligned chromosomes. The actin cytoskeleton was not affected (9). Five other compounds altered the mitotic spindle specifically; no effect was seen on microtubules, actin filaments, or chromatin in interphase cells. The mitotic phenotypes caused by these five small molecules included chromosome misalignment, loss of spindle pole organization, changes in spindle shape, and combinations of all three.

One of these five compounds was especially interesting. In BS-C-1 cells treated with this compound, the bipolar mitotic spindle (Fig. 2A) was replaced by a monoastral microtubule array surrounded by a ring of chromosomes (Fig. 2C). Interphase 
cells were not affected (Fig. 2, B and D). After 4 hours of treatment with this compound, a significant fraction of cells were arrested in mitosis and $90 \%$ of them displayed the monoastral phenotype. We therefore named this 1,4-dihydropyrimidine-based compound (Fig. 3D) monastrol (10)

Normal bipolar spindles are thought to assemble in part through interactions between antiparallel microtubules from the two halfspindles (Fig. 3A) (1/). Without these interactions the spindle remains monoastral (Fig. 3B), and the cell arrests in mitosis. Presumably the unattached kinetochores, the sites that allow microtubules to attach to chromosomes, generate signals that activate the mitotic checkpoint.

One class of proteins involved in the assembly and maintenance of the mitotic spindle is the family of mitotic kinesins, a subset of the kinesin superfamily. This superfamily contains over 100 proteins, whose other functions include organelle transport and membrane organization (12). The first evidence that mitotic kinesins are important in establishing spindle bipolarity came from genetic studies: temperaturesensitive mutants in the BimC family of kinesins do not form bipolar spindles at the restrictive temperature (13). Inhibition of the BimC kinesin Eg5 with Eg5-specific antibodies also induced monoasters similar to those observed after treatment with monastrol $(14,15)$. We therefore hypothesized that Eg5 might be a target of monastrol.

Like other kinesins, Eg5 can drive the movement of microtubules in vitro (16). Monastrol inhibited Eg5-driven microtubule motility (Fig. 3C) (17) with an $\mathrm{IC}_{50}$ (median inhibitory concentration) of 14 $\mu \mathrm{M}$ (Fig. $3 \mathrm{E}$ ). This is comparable to the EC (median effective concentration) of 22 $\mu \mathrm{M}$ observed in the cytoblot assay (9). Washout experiments demonstrated that the effect of monastrol is reversible in vitro (Fig. 3C). In vivo, essentially all cells that were arrested in monastrol for 4 hours completed mitosis after the compound was washed out (9). To test whether inhibition of microtubule motility is specific to monastrol, we tested the closely related compound DHP2 (Fig. 3D) (17). DHP2 did not arrest cells in mitosis or generate monoastral spindles, and it had no significant effect on Eg5-dependent microtubule motility in vitro (Fig. 3C).

To test whether monastrol affects other motor proteins, we first determined its ability to inhibit in vitro microtubule motility driven by conventional kinesin, the founding member of the kinesin superfamily (17). The $\mathrm{NH}_{2}$-terminal motor domain of conventional kinesin shares $33 \%$ sequence identity with the Eg5 motor domain (16). Monastrol $(200 \mu \mathrm{M})$ did not inhibit micro- tubule movement driven by conventional kinesin (Fig. 3F) (I8).

Several motor proteins, including conventional kinesin, are involved in the correct cellular localization of organelles such as Iysosomes or the Golgi apparatus. To test whether monastrol affects the activity of these motor proteins, we studied organelle localization in interphase cells treated with monastrol at concentrations three times the $\mathrm{EC}_{50}$ for mitotic arrest (8). Perturbation of any one of a number of these motor proteins would result in organelle mislocalization (19). No effect on the localization and organization of the Golgi apparatus or lysosomes was observed (Fig. 4). Thus, monastrol is not a general inhibitor of motor proteins.

Chromosomes attached to only one spindle pole display oscillatory movements away from and toward the spindle pole, reflecting the activity of kinetochore proteins, kinesins, and microtubule polymer-
A.

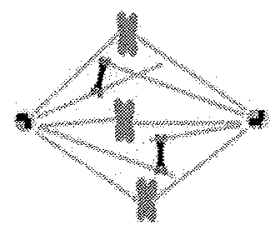

e.
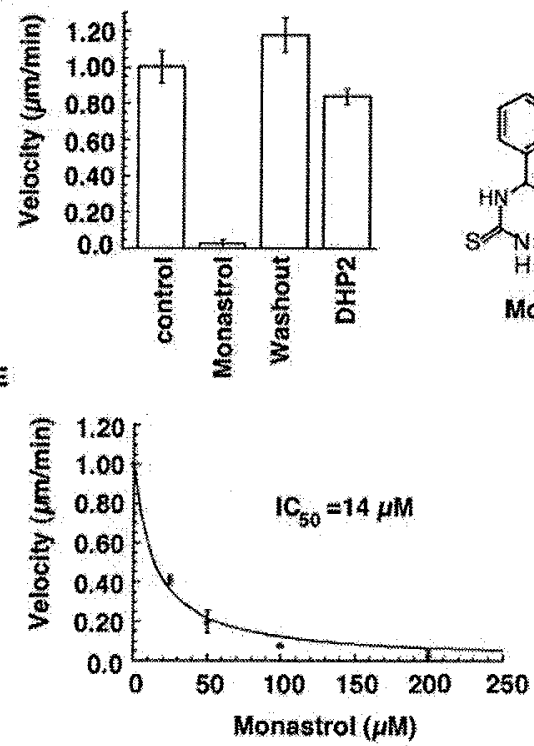

$\mathbf{D}$ ization dynamics (20). Images of live BS$\mathrm{C}-1$ cells treated with monastrol revealed that chromosomes did exhibit oscillatory behavior (Fig. 5) (8), indicating that monastrol does not inhibit chromosome attachment to microtubules.

The approach described here of screening for small molecules that affect a particular pathway or process, rather than a single protein activity, is referred to as chemical genetics because of its conceptual similarity to classic forward genetic screens $(21,22)$. The cytoblot assay is a particularly versatile tool for chemical genetics because it can provide a quantitative readout of essentially any posttranslational modification if appropriate antibodies are avail able. In this study, we used nucleolin phosphorylation as a readout of mitosis. We then used a cytological screen to study the phenotype of treated cells in more detail, which resulted in identification of a compound that targets a protein for which no
B

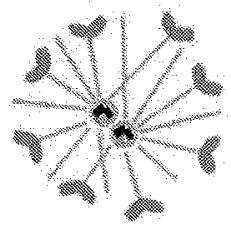<smiles>CCOC(=O)C1=C(C)NC(=S)NC1c1cccc(O)c1</smiles><smiles>CCOC(=O)C1=C(C)NC(=S)NC1c1ccc(O)cc1</smiles>

$+$

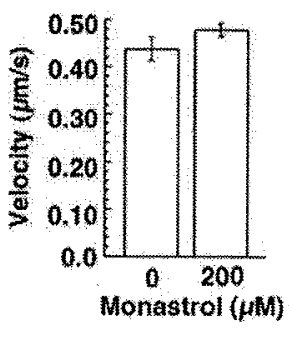

Fig. 3. Effects of monastrol can be explained by inhibition of Eg5 activity. (A) Model for spindle bipolarity. Plus-end-directed motors, such as Eg5, are thought to be involved in separation of the centrosomes and establishment of a symmetric spindle axis. Microtubules are shown in green, and chromosomes are blue. Eg5 (red) is depicted as a homotetramer (26). (B) Inhibition of kinesin Eg5 results in monoastral spindles $(14,15)$. (C) Monastrol reversibly inhibits microtubule motility driven by $\mathrm{Eg} 5$ in vitro. For the washout, we measured Eg5-driven microtubule motility in the presence of monastrol $(200 \mu \mathrm{M})$, and then we depleted the assay chamber of compound and immediately measured motility again. At $200 \mu \mathrm{M}$. DHP2, a related dihydropyrimidine, did not significantly inhibit Eg5-driven microtubule motility. Control, 2.5\% DMSO. (D) Chemical structures of monastrol and DHP2. (E) Monastrol inhibits the Eg5-driven microtubule motility with an $1 C_{50}$ of $14 \mu \mathrm{M}$. (F) Monastrol $(200 \mu \mathrm{M})$ does not inhibit microtubule motility driven by conventional kinesin. 

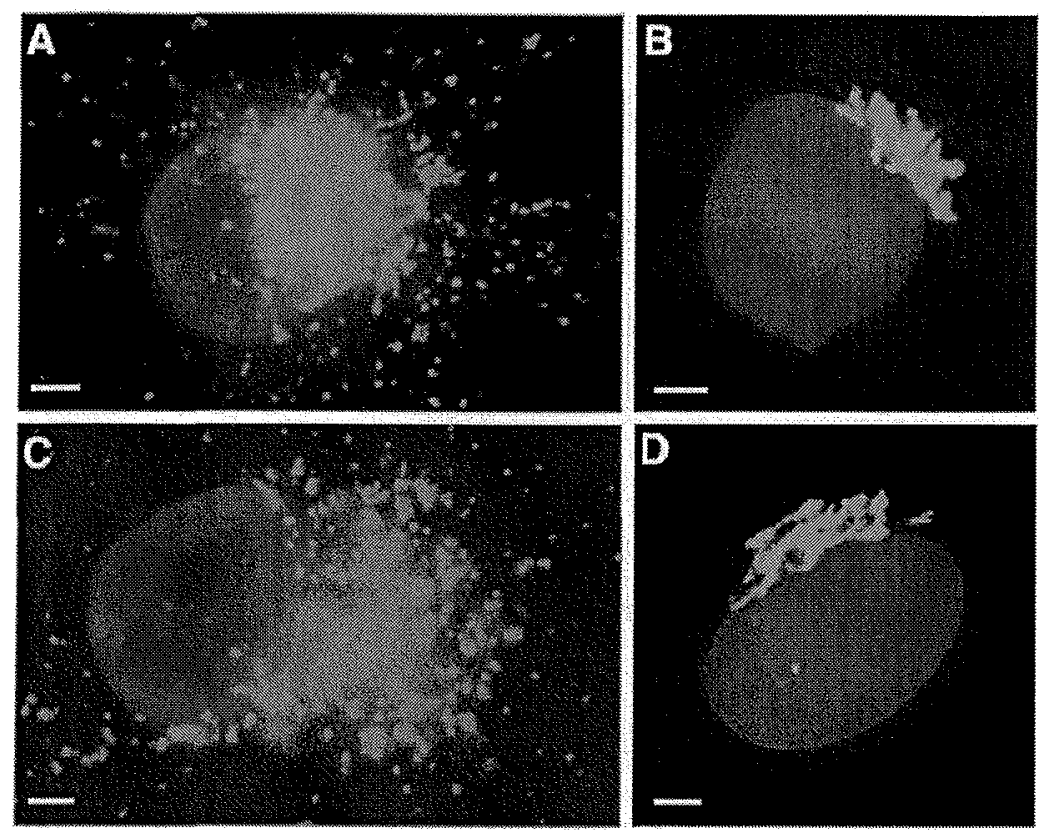

Fig. 4. Distribution of lysosomes and the Golgi apparatus is not affected by monastrol. We treated BS-C-1 cells for 4 hours with $0.4 \%$ DMSO (A and B) or $68 \mu \mathrm{M}$ monastrol ( $C$ and $D$ ) and then stained them for lysosomes ( $A$ and $C$ ) or Golgi apparatus ( $B$ and D) (8). Scale bars, $5 \mu \mathrm{m}$.
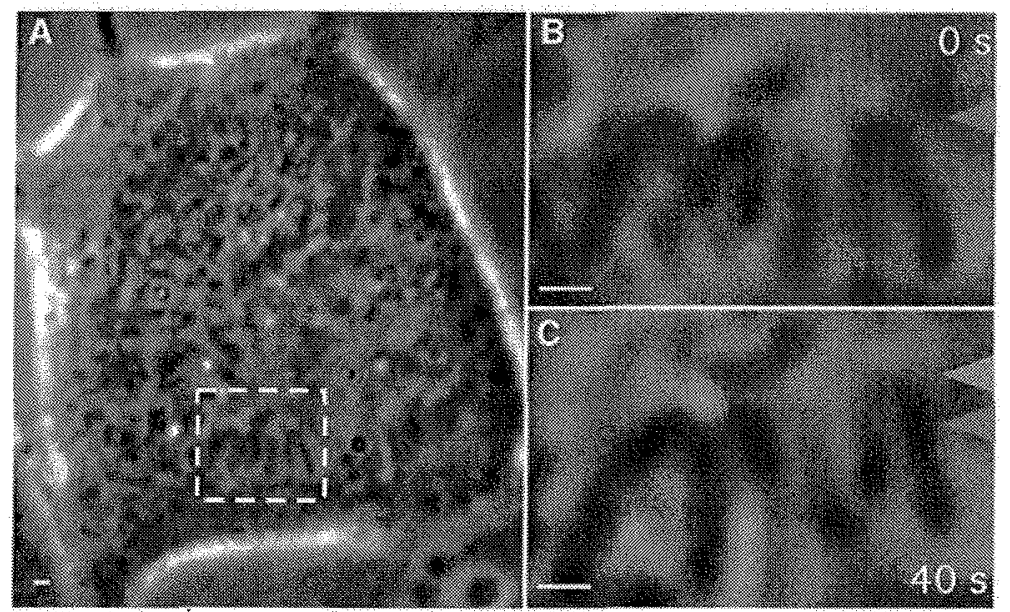

Fig. 5. Chromosomes surrounding monoastral spindles show oscillatory movement toward and away from the center of the aster. Time lapse images of a live BS-C-1 cell after treatment with 68 $\mu M$ monastrol. (A) Box marks the region enlarged in (B) and (C). Red and green arrowheads highlight position of a dynamic chromosome at two time points ( $B$ and $C$ ). The $V$ shape is characteristic of chromosomes that are attached to microtubules from only one pole. Scale bars, $1 \mu \mathrm{m}$.

cell-permeable small molecule modulator was previously known. The only kinesin inhibitors previously known are 5'-adenylylimido-diphosphate (AMP-PNP) (23) and a marine natural product (24). Neither is cell permeable, and both affect multiple kinesin family members. Because compounds that cause mitotic arrests by other mechanisms have shown antitumor activity in humans (25), monastrol may serve as a lead for the development of anticancer drugs. In any case, monastrol will be a valuable tool for dissecting the function of Eg5 in the establishment of spindle bipolarity and other

\section{References and Notes}

1. E. Hamel, Med. Res. Rev. 16, 207 (1996).

2. B. R. Stockwell, S. J. Haggarty, S. L. Schreiber, Chem. Biol. 6, 71 (1999)

3. H. J. Anderson et al., Exp. Cell. Res. 238, 498 (1998).

4. Library compounds (Diverset $E$, Chembridge Corporation) were tested at a final concentration of $-45 \mu \mathrm{M}$ cellular processes.
This library consists of a set of structurally diverse small molecules that vary in functional groups and charge. A549 (lung carcinoma) cells were treated with compound for 18 hours, then stained with a monoclonal antibody, TG-3, that recognizes a phosphorylated form of nucleolin (2). Cells in mitosis show a dramatic increase in TG-3 staining (3). We quantified TC3 binding by coupling secondary antibodies to an enzymatic luminescent reporter. We used the percentage of cells in mitosis after treatment with $0.1 \%$ dimethyl sulfoxide (DMSO) (control) to normalize the data.

5. Purification of bovine tubulin and labeling of tubulin with fluorescent dyes were performed as described [A. Desai et al., Cell 96, 69 (1999)]. We determined the critical concentration for microtubule polymerization [T. Mitchison and M. Kirschner, Nature 312, 237 (1984)] in the presence of each compound at $237(1984)$
$\sim 50 \mu \mathrm{M}$.

6. Supplementary material is available at wuw. sciencemag.org/feature/data/1043253.shl

7. S. J. Haggarty, unpublished data.

8. Images of tive BS-C-1 cells were taken as described [L. Cramer, T. Mitchison, J. Theriot, Curr. Opin. Cell Biol 6, 82 (1994)]. For immunofluorescence, we stained BS-C-1 cells with the Golgi-specific antibody (antiColgi 58K protein antibody) (Sigma) or with DM1 A, an antibody to $\alpha$-tubulin (Sigma). We used tetramethyl rhodamine isothiocyanate-conjugated phalloidin (Sigma) to visualize actin. Lysosomes were stained with LysoTracker (Molecular Probes). Images were obtained with a Nikon E-800 microscope or an Olympus IX70 inverted-microscope.

9. T. U. Mayer, data not shown

10. Monastrol used in all experiments after the screen was synthesized according to published protocols [K. Lewandowski et al., J. Comb. Chem. 1, 105 (1999)] and characterized by nudear magnetic resonance imaging and mass spectroscopy.

11. C. E. Walczak, I. Vernos, T. J. Mitchison, E. Karsenti, R. Heald, Curr. Biol. 8, 903 (1998).

12. R. D. Vale and R. J. Fletterick, Annu. Rev. Cell Dev. Biol. 13, 745 (1997).

13. A. P. Enos and N. R. Morris, Cell 60, 1019 (1990): I Hagan and M. Yanagida, Nature 356, 74 (1992); M. A. Hoyt et al.s. J. Cell Biol. 118, 109 (1992).

14. A. Blangy et at, Cell 83, 1159 (1995).

15. K. E. Sawin et al., Nature 359, 540 (1992)

16. T.M. Kapoor and T. J. Mitchison, Proc. Natl. Acad. Sci U.S.A. 96, 9106 (1999).

17. The plasmids encoding Xenopus Eg5 motor domain (residues 1 to 591) and human conventional kinesin (residues 1 to 560) have been described (16) [C. Woehlke et al., Cell 90, 207 (1997)]. Purification of Woehike et al., Cell 90, 207 (1997)]. Purification of
the expressed motor proteins and in vitro motility experiments were carried out as described (76). Monastrol and DHP2 were dissolved in DMSO (final concentration $2.5 \%$ ). All velocity data represent resutts from two or more independent experiments and the mean velocity is shown with the SE. Dose response data are best fit to hyperbola.

18. BS-C-1 cells treated with $200 \mu \mathrm{M}$ monastrol underwent cell lysis after a few hours. This is probably due to nonspecific cytotoxic effects.

19. N. Hirokawa, Science 279, 579 (1998).

20. C. L. Rieder et al., J. Cell Biol. 103, 581 (1986).

21. T. J. Mitchison, Chem. Biol. 1, 3 (1994)

22. S. L. Schreiber, Bioorg. Med. Chem. 6, 1127 (1998). 23. W. M. Saxton, Methods Cell Biol. 44, 279 (1994).

24. R. Sakowicz et al., Science 280, 292 (1998).

25. M. A. Jordan and L. Wilson, Methods Enzymol. 298 252 (1998).

26. C. E. Walczak et al., Curr. Biol. 8, 903 (1998).

27. The institute of Chemistry and Cell Biology is supported by NIH (CA78048) and Merck \& Co. This work was also supported by grants from the Human Frontier Science Program and National Institute of General Medical Sciences (39565). We thank T. Rapoport for the p58 antibody and the lysotracker dye, P. Davies for the TC3 antibody, and members of the Mitchison and Schreiber laboratory and R. Ward for comments on the manuscript. T.U.M. is a fellow of the Deutsche Forschungsgemeinschaft. T.M.K. is a Runyon-Winchell Fellow. 